\title{
Photoluminescence study of the effect of strain compensation on InAs/AlAsSb quantum dots
}

\author{
Zhexin Zhao, Ramesh B. Laghumavarapu, Paul J. Simmonds, Haiming Ji, Baolai Liang*, \\ and Diana L. Huffaker \\ California NanoSystems Institute \& Electrical Engineering Department, University of \\ California - Los Angeles, Los Angeles, California 90095, U.S.A.
}

\begin{abstract}
We investigate stacked structures of InAs/AlAsSb/InP quantum dots using temperatureand power-dependent photoluminescence. The band gap of InAs/AlAsSb QDs is $0.73 \mathrm{eV}$ at room temperature, which is close to the ideal case for intermediate band solar cells. As the number of quantum dot layers is increased, the photoluminescence undergoes a blue-shift due to the effects of accumulated compressive strain. This PL red shift can be counteracted using thin layers of AlAs to compensate the strain. We also derive thermal activation energies for this exotic quantum dot system.
\end{abstract}

Keywords: Photoluminescence, Quantum dots, Intermediate band solar cell, Activation energy

\section{Introduction}

Intermediate band solar cells (IBSCs) are among the most promising candidates for next generation photovoltaics, offering high efficiency from a simple structure at low cost. ${ }^{1,2}$ One possible method for creating the intermediate band is by inserting quantum dot (QD) layers into the active region of a solar cell. ${ }^{2,6-13}$ The band alignments of current QD material systems, such as InAs/GaAs, InAs/GaAsN and GaSb/GaAs, ${ }^{3-5}$ are not optimal for implementing the theoretically predicted intermediate band for IBSCs. In comparison with InAs/GaAs and GaSb/GaAs, the InAs/AlAsSb QD system has band alignments that almost match the calculated ideal case. ${ }^{6}$ Previous studies from our group show that this is a type-II QD system with strong electron confinement, that offers excellent optical characteristics for IBSCs. ${ }^{6}$ We have successfully optimized both the MBE growth conditions to obtain high-quality stacked QD structures, and QD optical performance to create IBSC prototype devices. ${ }^{6,7}$

So that QD IBSC devices can absorb solar energy more efficiently, it is important to obtain high QD density by stacking layers of dots. However, the compressive strain left over from the QD self-assembly process will also accumulate and cause defects in the stacked structure. It is therefore hard to achieve a large number of QD layers while maintaining high material quality. Strain compensation (SC) is a useful technique to deal with this problem ${ }^{8-12}$. We recently reported the use of thin tensile-strained AlAs layers to manage compressive strain in stacked layers of InAs/AlAsSb QDs. ${ }^{13}$ The AlAs SC layers enable us to reduce residual strain in the QD stacks, so improving the overall optical quality and increasing carrier lifetime.

In this work we report the results of comprehensive photoluminescence (PL) spectroscopy measurements to fully understand the fundamental characteristics of strain-compensated InAs/AlAsSb QDs. With a view to implementing this exotic QD system in proof-of-principle IBSC devices, we are particularly interested to learn how the material properties change with increasing number of stacked QD layers, and what role the SC layers play.

\section{Experiments}

We studied four InAs/AlAsSb QD samples grown on $\operatorname{InP}(001)$ substrates by solid-source 
molecular beam epitaxy. Schematic diagrams of the sample structure are shown in Fig. 1 and the sample details are listed in Table 1. All samples have the following structure: a $500 \mathrm{~nm}$ AlAsSb buffer, a 5 monolayer (ML) GaAs cladding layer, $8 \mathrm{ML}$ of InAs QDs, followed by a $5 \mathrm{ML}$ $\mathrm{GaAs}_{0.95} \mathrm{Sb}_{0.05}$ capping layer, ${ }^{7}$ and then a $15 \mathrm{~nm} \mathrm{AlAsSb}$ spacer. For sample B and sample $\mathrm{C}$ we repeated the whole QD section (including the GaAs cladding, the InAs QDs, the GaAsSb capping layer, and the AlAsSb spacer) 5 and 30 times, respectively. Sample D also has 30 QD layers, but with a 3 ML AlAs SC layer in the middle of each AlAsSb spacer. The 3ML AlAs SC layer has been carefully designed to fully balance the strain in the stacked QD structure. ${ }^{13}$ While the lattice constant of InAs is bigger than that of the InP substrate, the lattice constant of AlAs is smaller. The AlAs layers therefore introduce tensile strain that compensates the compressive strain caused by the InAs QDs in each period of the stack. We have previously reported the calculated band alignment for these strain compensated QD samples. ${ }^{13}$
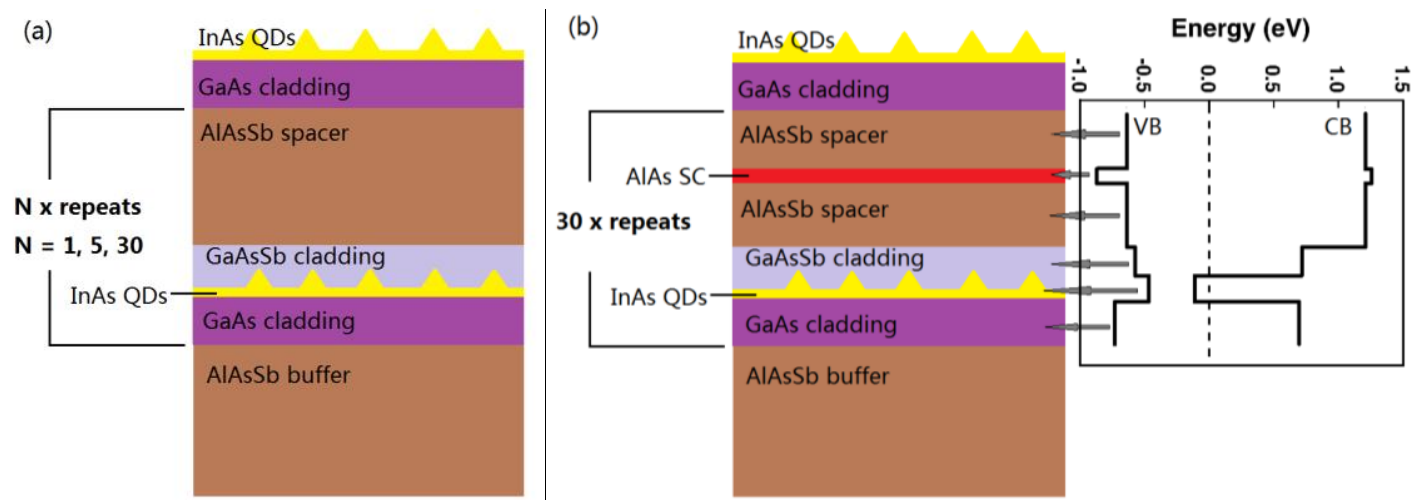

Fig. 1 Schematic diagram of InAs/AlAsSb QD samples (a) without, and (b) with an AlAs SC layer. The calculated band structure of the strain compensated sample is shown next to (b).

Table 1: Details of the four InAs/AlAsSb QD samples studied in this work

\begin{tabular}{lllll}
\hline \hline Sample ID & $\mathrm{A}$ & $\mathrm{B}$ & $\mathrm{C}$ & $\mathrm{D}$ \\
\hline \# of QD layers & 1 & 5 & 30 & 30 \\
Spacer thickness & -- & $15 \mathrm{~nm}$ & $15 \mathrm{~nm}$ & $15 \mathrm{~nm}$ \\
AlAs SC thickness & 0ML & OML & 0ML & $3 \mathrm{ML}$ \\
\hline \hline
\end{tabular}

For PL measurements, the samples are placed in a cryostat $(77-300 \mathrm{~K})$. A $532 \mathrm{~nm}$ solid-state laser operated at $8 \mathrm{~mW}, 25 \mathrm{~mW}$ and $80 \mathrm{~mW}$, is focused on the sample surface with a spot $\sim 125 \mu \mathrm{m}$ in diameter. The PL signal is collected by a lens and then dispersed by a monochromator. A Hamamatsu Extended-InGaAs detector is used to measure the PL signal via standard lock-in technology. The PL spectra are fitted with Gaussian functions in order to derive the PL peak wavelength, FWHM and integrated PL intensity (IPL).

\section{Experimental Results and Discussion}

The PL spectra of the four samples are normalized to the maximum intensity of each spectrum in Fig. 2(a). The PL peak blue-shifts from $1587 \mathrm{~nm}$ to $1459 \mathrm{~nm}$ as the number of stacked QD layers increases from 1 to 30 , while the 30-layer SC sample D emits at similar wavelength to the single QD layer sample A. The blue-shift from sample A to C may be attributed to the accumulated compressive strain, which tends to enlarge the band gap. However with the introduction of the SC layers in sample D, the amount of strain in each period of the QD stack remains constant. So, even though sample D has 30 QD layers, the use of strain compensation means that it emits at almost the same wavelength as the single layer sample A. This trend holds throughout the temperature range 
from $77 \mathrm{~K}$ to room temperature (Fig. 2(b)).
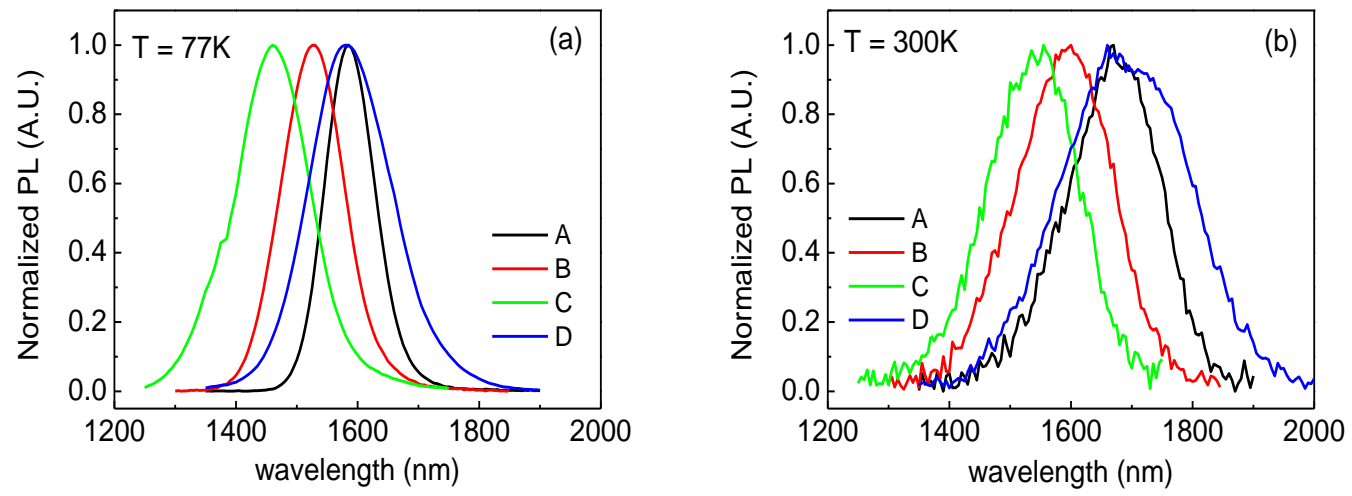

Fig. 2 (a) Normalized PL spectra measured with a laser power of $8 \mathrm{mWat} 77 \mathrm{~K}$. The PL intensities of samples B, C, and D are $0.74,1.02$ and 2.15 times the intensity of sample A, respectively. (b) Normalized PL spectra measured with a laser power of $25 \mathrm{~mW}$ at $300 \mathrm{~K}$. The PL intensities of samples B, C, and D are 1.38, 0.95 and 1.82 times the intensity of sample A, respectively.

Under the $8 \mathrm{~mW}$ excitation condition in Fig. 2 (a), sample D has the strongest PL with peak intensity twice that of the other three samples. The integrated PL intensities (normalized to that of sample A) of samples A, B, C and D at $77 \mathrm{~K}$ (with a laser power of $8 \mathrm{~mW}$ ) are found to be $1,0.93$, 1.43 and 3.48 respectively. Although increasing the number of layers of QDs may enhance light absorption, the simultaneous accumulation of strain also increases the occurrence of defects and deteriorates the optical quality. As a result, despite raising the number of stacked QD layers from 1 to 30 in samples A to C, the PL intensity remains essentially unchanged. However, in sample D the defects caused by excessive strain are reduced by the inclusion of the SC layers, and so the benefit of a large number of stacked QDs on the optical quality of the structure becomes apparent. We measure FWHM values of $82 \mathrm{~nm}, 103 \mathrm{~nm}$, and $118 \mathrm{~nm}$ for samples A, B, and C respectively. The broadening of the PL spectra with more QD layers may be due to the increasing inhomogeneity of QDs caused by stacking. It is also very interesting that the FWHM is $134 \mathrm{~nm}$ for sample D. Further work will be needed to understand why the PL of sample D with SC layers is broader than that of sample C without SC layers.

The PL as a function of temperature from $77-300 \mathrm{~K}$ is shown in Fig. 3. In sample D, thermal quenching starts at a relatively low temperature, which is a characteristic of type-II QDs. ${ }^{14}$ As the temperature increases, the PL peak energy shows a red shift for all samples due to the decrease of QD band gap predicted by the Varshni law, ${ }^{15}$

$$
E(T)=E(0)-\frac{\alpha T^{2}}{\beta+T}
$$

By fitting the temperature dependence of the peak PL energies using Equation (1), we can obtain the Varshni parameters $\alpha$, and $\beta$, while the transition energy at $0 \mathrm{~K}, \mathrm{E}(0)$, is offset in the four samples. Since in type-II QDs the PL peak energy is well known to depend on the excitation intensity, ${ }^{6,7,14}$ the fitting results also depend on the excitation intensity. Three different laser powers are thus used in the experiments to highlight this dependence and show the trend as excitation intensity approaches the solar power intensity $\left(\sim 1 \mathrm{~mW} / \mathrm{mm}^{2}\right)$. The Varshni fitting for sample D is shown in Fig. 3(b) as an example and the corresponding values for all four samples are listed in Table 2. These Varshni fitting results are satisfactory when compared to the values for bulk InAs 
recommended by Vurgaftman et al.: $\mathrm{E}(0)=0.417 \mathrm{eV}, \alpha=0.276 \mathrm{meV} / \mathrm{K}$, and $\beta=93 \mathrm{~K} .{ }^{15} \mathrm{In}$ our QD system, the transition energy $\mathrm{E}(0)$ is offset because of quantum confinement and the effect of strain on the band gap. Table 2 shows that increasing the number of QD layers raises $\mathrm{E}(0)$, while in sample D, inserting SC layers reduces E(0) to the value of the single QD layer sample A. Though $\alpha$ and $\beta$ are a little different from the literature values above, they are within a tolerable range and reveal some interesting phenomenon. $\alpha$ and $\beta$ change considerably from sample to sample, but the change is comparatively weak as a function of laser excitation power. Nevertheless, $\alpha$ for sample $\mathrm{C}$ is similar to that of sample D but differs significantly from samples A and B. We see the same trend for $\beta$. These results indicate that the number of stacked QD layers has stronger influence on $\alpha$ and $\beta$ than the introduction of SC layers, or the pump laser power. An investigation of parameters $\alpha$ and $\beta$ might therefore help reveal material properties that change as a result of stacking large numbers of QD layers.
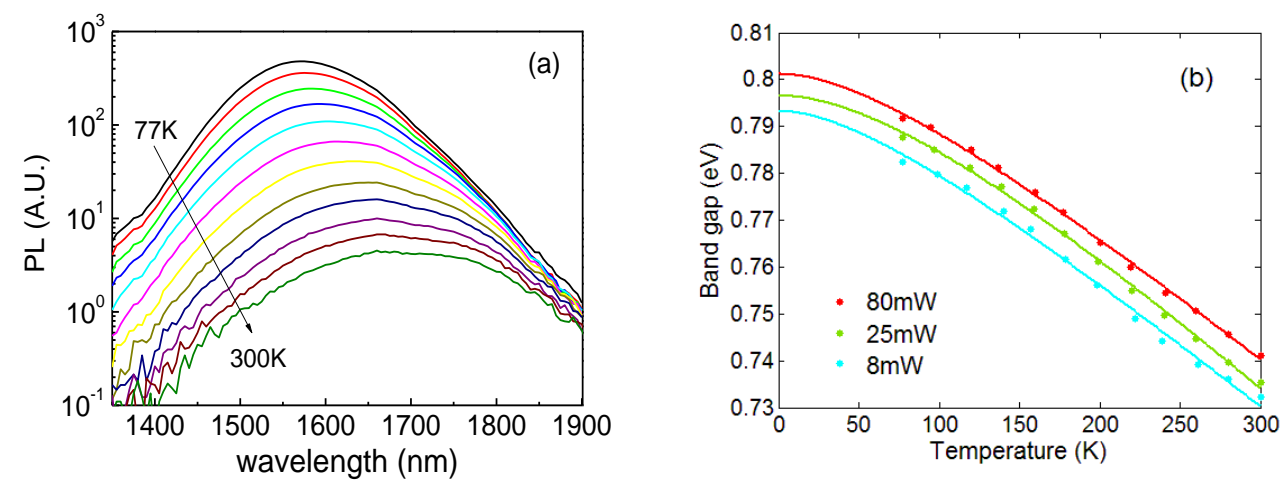

Fig. 3 (a) PL spectra of sample D measured at different temperatures from $77 \mathrm{~K}$ to $300 \mathrm{~K}$ using a laser power of $25 \mathrm{~mW}$; (b) Varshni fitting of the peak PL energies from the spectra in 2(a) at three different laser excitation powers.

Table 2: Varshni equation fitting results for all four samples as a function of pump laser power

\begin{tabular}{l|ccc|ccc}
\hline \hline Sample \# & \multicolumn{3}{|c|}{$\mathrm{A}$} & \multicolumn{3}{c}{$\mathrm{B}$} \\
\hline Laser power & $8 \mathrm{~mW}$ & $25 \mathrm{~mW}$ & $80 \mathrm{~mW}$ & $8 \mathrm{~mW}$ & $25 \mathrm{~mW}$ & $80 \mathrm{~mW}$ \\
$\mathrm{E}(0)(\mathrm{eV})$ & 0.790 & 0.796 & 0.805 & 0.822 & 0.828 & 0.835 \\
$\alpha(\mathrm{meV} / \mathrm{K})$ & 0.220 & 0.223 & 0.212 & 0.204 & 0.199 & 0.191 \\
$\beta(\mathrm{K})$ & 81 & 79 & 33 & 90 & 62 & 25 \\
\hline Sample \# & \multicolumn{3}{|c|}{$\mathrm{C}$} & & \multicolumn{3}{c}{$\mathrm{D}$} \\
\hline Laser power & $8 \mathrm{~mW}$ & $25 \mathrm{~mW}$ & $80 \mathrm{~mW}$ & $8 \mathrm{~mW}$ & $25 \mathrm{~mW}$ & $80 \mathrm{~mW}$ \\
$\mathrm{E}(0)(\mathrm{eV})$ & 0.858 & 0.861 & 0.864 & 0.793 & 0.796 & 0.801 \\
$\alpha(\mathrm{meV} / \mathrm{K})$ & 0.282 & 0.299 & 0.260 & 0.284 & 0.326 & 0.287 \\
$\beta(\mathrm{K})$ & 158 & 185 & 129 & 106 & 170 & 125 \\
\hline \hline
\end{tabular}

It is worth noting from Fig. 3(b) that at a laser power of $8 \mathrm{~mW}$ the peak PL energy for sample D is $0.73 \mathrm{eV}$ at $300 \mathrm{~K}$. This value is very close to the optimal value $(\sim 0.7 \mathrm{eV})$ for an IBSC. ${ }^{1}$ IBSC theory predicts that only when the band alignment is close to the optimal case can the theoretical maximum power conversion efficiency be achieved. The careful design of the InAs/AlAsSb QD system for achieving highly efficient IBSCs is further confirmed by PL emission from the single QD layer sample A at $0.74 \mathrm{eV}$ at $300 \mathrm{~K}$. The peak PL energies for samples B and C are $0.78 \mathrm{eV}$, and $0.81 \mathrm{eV}$ respectively at $300 \mathrm{~K}$ and the deviation from the optimal value grows larger with more QD layers. However, this deviation is eliminated when SC is applied to the QD system. The use of SC layers guarantees that the band alignment is still close to the optimal value, even when the number 
of QD layers is large, as it will need to be in a practical IBSC device.

Thermal activation energy, another critical parameter for carriers in a QD system, refers to the energy that the carriers need to escape from the QDs. The integrated PL intensity (IPL) is related to thermal activation energy $\left(\mathrm{E}_{\mathrm{a}}\right)$ by, ${ }^{16}$

$$
I P L \propto \exp (E a / k T)
$$

We derive the thermal activation energy of the QDs from the thermal quenching processes close to room temperature. Fig. 4 shows the Arrhenius plot of IPL as a function of measurement temperature at three different pump powers - the example shown here is for sample D. The gradients of the linear fits at high temperature give the thermal activation energies and these are summarized for all four samples in Table 3. Thermal activation energy depends on the excitation power density: higher excitation power leads to a lower measured activation energy. At the same power density, the thermal activation energy does not vary much between samples, except that sample $\mathrm{C}$ appears to be an outlier, showing higher thermal activation energies at all pump powers compared with the other three.

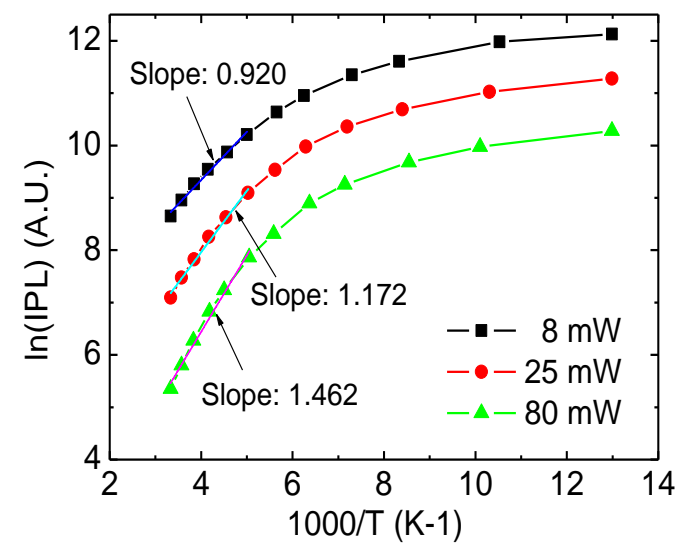

Fig. 4. Linear fitting of $\ln ($ IPL) against $1000 / \mathrm{T}$ at high temperature for sample $\mathrm{D}$. The slopes are annotated near the linear fitting.

Table 3. Thermal activation energies for the four samples at different excitation laser powers.

\begin{tabular}{lccc}
\hline \hline Activation Energy (meV) & $8 \mathrm{~mW}$ & $25 \mathrm{~mW}$ & $80 \mathrm{~mW}$ \\
\hline Sample A & 133 & 99 & 73 \\
Sample B & 130 & 102 & 74 \\
Sample C & 160 & 128 & 100 \\
Sample D & 126 & 101 & 79 \\
\hline \hline
\end{tabular}

From the data in Table 3, we note that the activation energy of InAs/AlAsSb QDs decreases with increasing excitation laser power. Similar observations are reported by Le Ru et al. for the InAs/GaAs QD system. ${ }^{17}$ If we can apply their model to the InAs/AlAsSb QD system, the average number of carriers captured in every QD may have an impact on the experimentally derived thermal activation energy. As the InAs/AlAsSb QD system has a type-II band alignment, after excitation, electrons will be confined in the QDs, with holes localized outside but close to the QDs. With stronger excitation laser power, on average every QD has more electrons in it and more holes near it. This leads to a distortion of the band structure, reducing the energy required by the carriers to escape the QDs.

To explain the inconsistency of sample $\mathrm{C}$ with the others in Table 3, we must consider that fact that it contains the highest number of stacked QD periods but no SC layers. As such, it is likely to 
have the highest defect density and hence a shorter carrier lifetime. ${ }^{13}$ As a result, for a given pump laser power, each QD in sample $\mathrm{C}$ may, on average, have fewer carriers than the other three samples. The distortion of the band structure will therefore be less in sample $\mathrm{C}$ and so the measured activation energies are larger.

\section{Conclusions}

We have studied the photoluminescence response of InAs/AlAsSb QD structures. We see that stacking multiple layers of QDs leads to an accumulation of compressive strain, which increases the band gap and causes a blue-shift in the PL emission energy. Placing a carefully optimized tensile AlAs strain compensation layer in the AlAsSb spacer between each layer of QDs effectively balances the strain and improves the optical properties of the InAs dots. Through temperature and excitation power dependent PL measurements, we obtain Varshni parameters, as well as the thermal activation energies. We use these values to help us understand the carrier dynamics and the physical properties of these materials. The results presented here show that the strain compensated stacked InAs/AlAsSb QD system is a promising candidate with almost ideal band alignments for developing a high efficiency QD IBSC.

Acknowledgement: This project is supported by the US Department of Energy (through Grant number DE-EE0005325), and the Cross-disciplinary Scholars in Science and Technology (CSST) program between University of California, Los Angeles and Tsinghua University.

\section{References}

${ }^{1}$ A. Luque and A. Martí, Phys. Rev. Lett. 78, 5014 (1997).

${ }^{2}$ A. Luque and A. Martí, Advanced Materials22, 160 (2010)

${ }^{3}$ H. Jiang and J. Singh, Phys. Rev. B 56, 4696 (1997).

${ }^{4}$ F. Hatami, N.N. Ledentsov, M. Grundmann, J. Böhrer, F. Heinrichsdorff, M. Beer, D. Bimberg, S.S. Ruvimov, P. Werner, U. Gösele, J. Heydenreich, U. Richter, S. V. Ivanov, B.Y. Meltser, P.S. Kop'ev, and Z.I. Alferov, Appl. Phys. Lett. 67, 656 (1995).

${ }^{5}$ F. Hatami, M. Grundmann, N.N. Ledentsov, F. Heinrichsdorff, R. Heitz, J. Böhrer, D. Bimberg, S.S. Ruvimov, P. Werner, V.M. Ustinov, P.S. Kop'ev, and Z.I. Alferov, Phys. Rev. B 57, 4635 (1998).

${ }^{6}$ P.J. Simmonds, R.B. Laghumavarapu, M. Sun, A. Lin, C.J. Reyner, B. Liang, and D.L. Huffaker, Appl. Phys. Lett. 100, 243108 (2012).

${ }^{7}$ M. Sun, P.J. Simmonds, R.B. Laghumavarapu, A. Lin, C.J. Reyner, H.-S. Duan, B. Liang, and D.L. Huffaker, Appl. Phys. Lett. 102, 023107 (2013).

${ }^{8}$ P. Lever, H. H. Tan, and C. Jagadish, J. Appl. Phys. 95,(2004). 
${ }^{9}$ R. B. Laghumavarapu, M. El-Emawy, N. Nuntawong, A. Moscho, L. F. Lester, and D. L. Huffaker, Appl. Phys. Lett. 91, 243115 (2007)

${ }^{10}$ Ryuji Oshima,Ayami Takata, and Yoshitaka Okada, Appl. Phys. Lett, 93, 083111 (2008).

${ }^{11}$ Christopher G. Bailey, David V. Forbes, Ryne P. Raffaelle and Seth M. Hubbard, Appl. Phys. Lett. 98, 163105 (2011).

${ }^{12}$ C.G. Bailey, D.V. Forbes, S.J. Polly, Z.S. Bittner, Y. Dai, C. Mackos, R.P. Raffaelle, and S.M. Hubbard, IEEE Journal of Photovoltaics 2, 269 (2012)

${ }^{13}$ P.J. Simmonds, M. Sun, R.B. Laghumavarapu, B. Liang, A.G. Norman, J.-W. Luo, and D.L. Huffaker, Nanotechnology 25, 445402 (2014).

${ }^{14}$ K. Suzuki, R.A. Hogg, and Y. Arakawa, J. Appl. Phys. 85, 8349 (1999).

${ }^{15}$ I. Vurgaftman, J.R. Meyer, and L.R. Ram-Mohan, J. Appl. Phys. 89, 5815 (2001).

${ }^{16}$ Z. Xu, Z. Lu, X. Yang, Z. Yuan, B. Zheng, J. Xu, W. Ge, Y. Wang, J. Wang, and L. Chang, Phys. Rev. B 54, 11528 (1996).

${ }^{17}$ E. Le Ru, J. Fack, and R. Murray, Phys. Rev. B 67, 245318 (2003). 